\section{Auszeichnung für Asthmaforschung}

Das Remodeling beim Asthma bronchiale trägt wesentlich zur kontinuierlichen Abnahme der Lungenfunktion bei. Die zugrundeliegenden pathogenetischen Prozesse sind noch weitgehend unklar, aber ein wichtiger Ansatzpunkt für eine noch effektivere Therapie. Von großer pathogenetischer Bedeutung scheint die Akkumulation von Fibroblasten in der Lunge zu sein, die unter anderem die Synthese und Sekretion extrazellulärer Matrixproteine induzieren. Dr. Tillie-Louise Hackett vom St. Paul's Hospital an der BritishColumbia Universität in Vancouver ist jetzt der Nachweis gelungen, dass sich mit TGF- $\beta 1$ in Lungenepithelzellen eine epitheliale-mesenchymale Transition (EMT) induzieren lässt. Durch eine EMT wird die Zahl der Fibroblasten im Lungengewebe erhöht und damit möglicherweise das Remodeling vorangetrieben. Die Wissenschaftlerin wurde mit dem International Klosterfrau Award for Research Airway Diseases in Childhood 2011 ausgezeichnet. Der Preis wurde anlässlich der 33. Tagung der Gesellschaft für Pädiatrische Pneumologie im April 2011 in Graz, Österreich, verliehen und ist mit 30000 Euro dotiert.

Pressemeldung von Klosterfrau, 2.4.2011

\section{Atemwegstherapie mit neuem Auftritt}

Mit dem lyophilisierten, normierten Bakterienextrakt Broncho-Vaxom ${ }^{\circledR}$ steht ein Immuntherapeutikum zur Verfügung, das eingesetzt werden kann, um rezidivierende Infektionen der oberen und unteren Atemwege, besonders infolge chronischer Erkrankungen wie z. B. Bronchitis oder Sinusitis, vorzubeugen oder zu mildern. Falls eine Antibiotikatherapie notwendig ist, sollte Broncho-Vaxom ${ }^{\circledR}$ von Beginn an damit kombiniert werden. Bei Behandlungsbeginn im Infektionsschub wird die Einnahme über mindestens einen Monat empfohlen. Ab sofort ist Broncho-Vaxom ${ }^{\circledast}$ in einem neuen Packungsdesign erhältlich. Der Vertrieb wurde 2010 von der Vifor Pharma Deutschland übernommen. BronchoVaxom $^{\oplus}$ ist in der Akut- und Langzeittherapie einsetzbar und kann für Erwachsene als 7-mg-Kapseln und für Kinder als 3,5-mgKapseln oder als Granulat verschrieben werden. Weitere Informationen sind unter der E-Mail: info-de@viforpharma.com erhältlich.

PNEUMONEWS

\title{
Herausgeber Klinik:
}

Prof. Dr. A. Gillissen, Kassel

Prof. Dr. M. J. Kohlhäufl, Stuttgart-

Gerlingen

\section{Schriftleiter:}

Priv.-Doz. Dr. S. Budweiser, Rosenheim (Beatmungsmedizin)

Prof. Dr. G. Friedel, Stuttgart-Gerlingen (Thoraxchirurgie)

Prof. Dr. A. Ghofrani, Gießen, Dr. K. Gutjahr, Leipzig (Erkrankungen des Lungenkreislaufs)

Prof. Dr. A. Gillissen, Kassel (Asthma bronchiale)

Prof. Dr. M. Griese, München (Pädiatrische Pneumologie)

Prof. Dr. A. Günther, Prof. Dr. Ph. Markart, Gießen (Interstitielle Lungenerkrankungen)

Prof. Dr. M. J. Kohlhäufl, Stuttgart-Gerlingen (Tumoren)

Dr. S. R. Ott, Bern (Infektiologie)

Prof. Dr. K. Rasche, Wuppertal, Prof. Dr. K.-H. Rühle, Hagen, Prof. Dr. H. Schäfer,

Völklingen (Schlafmedizin)

Prof. Dr. G. Rohde, Maastricht/Niederlande (COPD, Emphysem)

Dr. K. Schultz, Bad Reichenhall (Rehabilitation)

Prof. Dr. M. Schwab, Prof. Dr. K. Mörike, Tübingen (Pharmakologische Therapie)

\section{Redaktion:}

Dr. Beate Schumacher (Chefredaktion, verantwortlich, Anschrift wie Verlag, beate.schumacher@springer.com) Ute Kempf (ute.kempf@springer.com), Dr. Judith Neumaier (judith.neumaier@t-online.de), Dr. Thomas Riedel, Angelika Holzgreve (Assistenz Chefredaktion, angelika.holzgreve@ springer.com)

\section{Verlag:}

Springer Medizin, ๑ Urban \& Vogel $\mathrm{GmbH}$, Aschauer Str. 30, D-81549 München, Tel.: 0 89/20 30 43-13 00, Fax: -13 99, http://www.springerfachmedien-medizin.de. Springer Medizin ist Teil der Fachverlagsgruppe Springer Science+Business Media.

\section{Geschäftsführer:}

Harm van Maanen, Ulrich Huber, Stephan Kröck, Dr. med. Esther Wieland, Matthias Wissel.

\section{Anzeigenverkauf:}

Ines Spankau (Anzeigenleitung, -1339, verantwortlich, Anschrift wie Verlag), Anzeigenpreisliste Nr. 4 vom 1. Oktober 2010.

Corporate Publishing:

Ulrike Hafner (06221/4878104).

\section{Herstellung}

Alison Hepper (Leitung); Schmidt Media Design München (Layout);

Dieter L. Adam, München (Titelbild)

\section{Vertrieb/Marketing Services:}

Frank Niemann (Leitung, -14 11)

\section{Abonnement:}

Die Zeitschrift erscheint 6 x jährlich. Bezugspreise ab 2011: Einzelheft $12 €$ inkl. der deutschen MwSt. zuzügl. 2,50 € Versandkosten; Jahresabo $75 €$; Studenten/Ärzte in Aus- und Weiterbildung $60 €$, jeweils zuzügl. Versand (Inland $21 €$, Ausland $36 €$ ).

Bestellungen nimmt der Verlag und jede Buchhandlung entgegen. Das Abonnement verlängert sich jeweils um ein Jahr, wenn es nicht zwei Monate vor Ende des Bezugszeitraums abbestellt wurde.

\section{Abonnentenservice:}

Tel.: 062 21/3 45-43 04; Fax: 062 21/3 45-42 29

Gesamtherstellung: Stürtz GmbH, AlfredNobel-Str. 33, D-97080 Würzburg

Originalien: Zur Veröffentlichung kommen nur Arbeiten, die an anderer Stelle weder angeboten noch erschienen sind. Die Autoren sind verpflichtet zu prüfen, ob Urheberrechte Dritter berührt werden. Alle CME-Manuskripte werden von unabhängigen Experten begutachtet. Eine redaktionelle Bearbeitung bleibt vorbehalten. Autoren, die mit vollem Namen genannt sind, veröffentlichen ihre Beiträge in alleiniger Verantwortung.

Copyright: Der Verlag behält sich das ausschließliche Recht der Verbreitung, Übersetzung und jeglicher Wiedergabe auch von Teilen dieser Zeitschrift durch Nachdruck, Fotokopie, Mikrofilm, EDV-Verwertung, Funk- oder Fernsehaufzeichnung vor. Jede gewerblich hergestellte oder benutzte Fotokopie verpflichtet nach $\S 54$ (2) UrHG zur Gebührenzahlung an die VG Wort, Abt. Wissenschaft, Goethestr. 49, D-80336 München, von der die Modalitäten zu erfragen sind.

Rechtseinräumung der Autoren: Mit der Einsendung eines Manuskripts zur Veröffentlichung überträgt der Verfasser dem Verlag für den Fall der Annahme das Recht, das Manuskript geändert oder unverändert, ganz oder teilweise in Pneumo-News und anderen Publikationen der Fachverlagsgruppe, in den zugehörigen Onlinediensten, in Onlinedatenbanken Dritter und, soweit vereinbart, gegen Nachhonorar in Sonderdrucken für Industriekunden zu nutzen.

Hinweis: Die in dieser Zeitschrift angegebe-nen Dosierungen - vor allem von Neuzulassungen sollten in jedem Fall mit dem Beipackzettel der verwendeten Medikamente verglichen werden. ISSN 1865-5467 